\title{
Jacques Ellul et le phénomène moral : universalité du besoin de justification, singularité de l'éthique chrétienne
}

Pierre Prades

\section{(2) OpenEdition \\ Journals}

Édition électronique

URL : http://journals.openedition.org/assr/26294

DOI : $10.4000 /$ assr.26294

ISSN : $1777-5825$

Éditeur

Éditions de l'EHESS

\section{Édition imprimée}

Date de publication : 31 décembre 2014

Pagination : 55-70

ISBN : $978-2-7132-2467-6$

ISSN : 0335-5985

Référence électronique

Pierre Prades, « Jacques Ellul et le phénomène moral : universalité du besoin de justification,

singularité de l'éthique chrétienne », Archives de sciences sociales des religions [En ligne], 168 | 2014,

mis en ligne le 30 mars 2018, consulté le 19 avril 2019. URL : http://journals.openedition.org/ assr/26294; DOI : 10.4000/assr.26294

Ce document a été généré automatiquement le 19 avril 2019.

(C) Archives de sciences sociales des religions 


\title{
Jacques Ellul et le phénomène moral : universalité du besoin de justification, singularité de l'éthique chrétienne
}

\author{
Pierre Prades
}

\section{RÉFÉRENCE}

ELLUL Jacques, Le Vouloir et le Faire. Une critique théologique de la morale, Genève, Labor et Fides, « Hors collection », réédition, 2013, 312 p.

1 Les étudiants bordelais qui, dans les années 1970, écoutaient d'une oreille pas toujours attentive les cours de Jacques Ellul (1912-1994) à l'IEP ou à la faculté de Droit, avaient conscience d'être face à un professeur hors normes, mais sans bien prendre sa vraie mesure. On savait qu'un contingent d'étudiants californiens venait chaque année passer un semestre à Bordeaux pour l'entendre, mais on ne comprenait pas bien pourquoi... Il enseignait aussi bien l'Histoire des institutions, sa discipline officielle, que la pensée de Marx, et il était déjà connu pour sa critique de la société technicienne. Engagé sur les terrains humanitaire et écologique, mais critique de l'engagement politique, fervent lecteur et admirateur de Marx mais sévère avec le marxisme, il était en empathie distante avec les jeunes contestataires de 68 tout en pensant radicalement contre son époque, donc aussi contre eux. Il accueillait d'un regard malicieux de ses yeux plissés les élans militants de ceux qui partageaient son intérêt pour la pensée de Marx, mais il refroidissait aussitôt leur ardeur d'une mise au point brève et sans appel, adoucie par une voix teintée d'un léger accent gascon et empreinte d'une évidente bienveillance.

2 Non seulement Jacques Ellul apparaissait en déphasage complet avec les courants dominants de l'époque, mais la plupart de ses étudiants ignoraient alors presque totalement l'autre partie de son activité intellectuelle et le dialogue serré qu'il 
poursuivait avec les philosophes et les théologiens les plus éminents du siècle, lui qui n'était ni l'un ni l'autre (il s'en justifie p. 20). Certes, il était une figure notoire du protestantisme en France, et il ne manquait pas lui-même d'en reconnaître l'inspiration. Mais parmi ceux qui pouvaient être intéressés par sadouble critique du capitalisme et du socialisme comme deux figures de la société technicienne, bien peu auraient eu l'idée d'ouvrir ce livre, paru en 1964 et réédité récemment par Labor et Fides : Le Vouloir et le Faire. Une critique théologique de la morale.

3 Comme le rappellent Denis Müller et Frédéric Rognon, dans leur introduction à cette nouvelle édition, ce livre est à considérer comme le premier élément d'une trilogie éthique, en rapport dialectique avec l'autre volet de l'œuvre d'Ellul, longtemps le plus connu, sa critique sociologique. Le Vouloir et le Faire (1964), Éthique de la Liberté (1975), Les combats de la liberté (1984), feraient ainsi pendant à La technique ou l'enjeu du siècle (1954), Le système technicien (1977), Le bluff technologique (1988). Cependant, les deux auteurs avertissent aussi que Jacques Ellul n'a pas eu le temps de mener à bien son projet, une " pentalogie » complétée par une Éthique de la sainteté, qui paraitra peut-être un jour, et une Éthique de l'amour, qu'il n'a pu commencer. «En conséquence, Le Vouloir et le Faire doit être lu comme la porte d'entrée d'un édifice dont les fondations avaient déjà été vigoureusement posées, mais dont le dernier étage ne sera jamais construit » (p. 8). Ellul voyait l'ensemble de son œuvre - une cinquantaine d'ouvrages, des centaines d'articles comme un seul grand livre dans lequel se répondent les deux courants de son inspiration, la critique de Marx et la Révélation de l'Évangile. La connaissance que nous pouvons avoir de cette dialectique, avec le recul du temps, permet de mieux comprendre aujourd'hui ce qu'était la position si étrange et paradoxale d'Ellul dans la France de son époque : celle du dissenter, une figure peu familière dans un pays dont la tradition catholique s'est sécularisée en un socialisme aux contours incertains, mais qui a eu un rôle essentiel dans l'évolution du monde protestant. Le ressort critique du dissent est tendu entre deux convictions, celle de la nécessité de la Parole du Christ, et celle de l'impossibilité qu'elle soit vraiment entendue par une humanité déchue. Qu'une même proposition soit à la fois nécessaire et impossible, c'est ce que Jacques Ellul articule dans ce livre à propos d'une «morale chrétienne », mais c'est peut-être aussi ce qui met en tension « dialectique » les deux volets de son œuvre, à partir du postulat de la discontinuité absolue entre l'histoire des hommes et le Royaume de Dieu (p. 28-31).

Ainsi, lorsqu'il affirme la nécessité pour les hommes de définir eux-mêmes une morale qui puisse traduire en exigences éthiques ce qu'ils définissent "librement» comme le Bien et le Mal - puisque la Chute est une accession à l'autonomie - il en tire logiquement la conséquence de la relativité de la morale, chaque groupe humain répondant à cette nécessité dans les conditions historiques qui lui sont propres. Il traque aussi sans concessions les tendances christianocentriques et européocentriques qui ont conduit les Occidentaux à se croire porteurs d'une morale universelle, c'est-à-dire la plus proche de la morale «naturelle». Ellul peut alors apparaître comme un compagnon de route des anthropologues et des sociologues les plus critiques de la domination impérialiste occidentale - bien qu'il n'emploie ni leurs concepts ni leur langage - de même qu'il a pu sembler en résonance avec les anticapitalistes dans sa critique de la société technicienne qui érige l'efficacité utilitariste en valeur suprême. Mais il va tout aussi loin dans un autre sens, en affirmant que cette critique, si elle peut éclairer l'humanité sur la réalité de sa condition, ne suffira jamais à donner un sens à sa vie, ni à la faire progresser. Il diverge 
ainsi de tous les « progressismes », tant dans l'ordre du politico-social que dans celui de la morale, du «bien agir ».

Ellul reprend ainsi un « parallélisme » caractéristique de la tradition réformée : aussi loin que puisse aller l'homme «naturel » dans son expérience humaine, il n'accédera pas par celle-ci à la dimension "spirituelle» de son humanité. Celle-ci ne peut que lui être " révélée », marquée dans son cœur par le Saint-Esprit, et les conséquences sur sa vie sont d'un tout autre ordre que celles qu'il tire de son expérience. Si l'humanité dans son ensemble, qu'elle soit chrétienne ou pas, ne peut que s'efforcer d'être "morale » de diverses façons, le chrétien quant à lui est appelé à agir au-dessus - et parfois contre cette morale établie de son temps, pour accomplir ce qu'il croit être les commandements divins.

\section{La morale comme nécessité pour l'homme déchu}

Il consacre ainsi la première partie de son livre à montrer que la morale est une affaire purement humaine, et qu'il est vain de chercher à légitimer, à justifier une position morale en s'inspirant de la Révélation divine : celle-ci nous enjoint de vouloir et de faire le Bien, sans nous dire en quoi il consiste, car l'homme ne connaît pas plus le contenu du Bien après sa chute qu'avant celle-ci. Admettre le contraire serait limiter la liberté de Dieu. Son point de départ, comme on l'a déjà signalé, est que la Chute est une prise d'autonomie. Devenus " comme des dieux ", les hommes ont à définir eux-mêmes le Bien et la Morale. Le "phénomène moral » est le fruit de cette condition, et ne peut donc dépasser l'ordre du péché. Ellul conteste ainsi le protestantisme libéral comme le catholicisme social, qui nient la discontinuité entre l'histoire humaine et le Royaume divin et affirment la perfectibilité de l'homme, individuelle ou collective.

7 Si la morale est une nécessité pour l'homme déchu, qui ne suit plus la volonté de Dieu, l'éthique chrétienne est d'une autre nature: elle relève de la liberté, qui est paradoxalement un renoncement à l'autonomie, une désaliénation, une libération de soimême pour devenir disponible à l'action selon la volonté divine. Mais alors cette éthique ne peut se fonder que sur la Révélation : si la morale est entièrement humaine, l'éthique chrétienne cherche une inspiration divine au-dessus de la morale. Elle ne se substitue pas aux morales humaines mais crée une tension avec elles: de la foi découle une exigence, celle d'accomplir les commandements, non pas au nom d'un idéal, d'un «Bien » défini par les hommes, mais pour proclamer et sceller une alliance. Si donc la morale humaineest une nécessité - historique, sociale, psychologique - l'éthique chrétienne en est disjointe, elle est une obligation volontaire que produit la foi - la pistis des Évangiles.

\section{Le phénomène moral et la diversité des morales du monde}

Ellul propose alors une anthropologie des morales humaines, dans sa deuxième partie «Morale du monde ». Le phénomène moral dont il veut rendre compte est à la fois objectif et subjectif, collectif et individuel, il inclut les morales théoriques et "sociologiques", celles-ci seules ayant une portée pratique, mais aussi la coutume morale, les impératifs de conscience et les choix éthiques personnels (p.137-140). Le phénomène moral serait, dans le langage de Marcel Mauss, un fait total. Et c'est ce 
qu'exprime la grande diversité des morales sociologiques, chacune liée à des conditions particulières de l'expérience humaine. La recherche de la morale "naturelle » au-delà d'une diversité superficielle des morales est elle-même un leurre, car elle est inspirée par des conditions historiques singulières: l'idée d'une morale universelle est d'origine chrétienne, elle est elle-même construite sur une vision universaliste du monde (p. 149). C'est de ce même point de vue critique qu'Ellul considère les «morales théoriques ", tel l'existentialisme- l'ouvrage paraît, rappelons-le, en 1964 - comme des phénomènes locaux et temporaires, des tentatives de rationaliser les tendances d'une société (p. 157-161).

9 Après cette relativité du phénomène moral, Ellul insiste sur les conditions d'applicabilité d'une morale : la relation entre morale théorique et morale sociologique. Si la première est l'expression construite de conditions historiques et sociales, elle peut néanmoins, en retour, influencer les conduites et renouveler la seconde (p. 162). Mais à condition de pouvoir être interprétée, adaptée, déformée, de subir une « adultération » dans un code éthique. Cette condition est à la fois psychologique, sociale et politique: comme le propose Bergson dans Les deux sources de la religion et de la morale, une morale créatrice doit pouvoir s'appuyer sur une émotion, sur une passion qu'elle cristallise en représentations, et s'incarner dans une figure exemplaire (p. 161, n. 1). Elle devient une force collective permanente lorsqu'elle est proclamée comme orthodoxie par les classes dirigeantes et l'État (p. 162).

Le phénomène moral se présente donc comme l'articulation entre un ensemble de représentations - un système de valeurs hiérarchisé autour d'un « motif central » - et des structures concrètes - psychologiques, sociales, politiques, religieuses. Une crise morale apparait lorsque cette articulation entre système de valeurs et structures concrètes s'affaiblit: ces dernières ne sont plus ressenties comme nécessaires, ou bien le motif central n'est plus reconnu comme tel par la société (p.189). Cette dynamique du phénomène moral s'exprime par un lien entre valeur et sacré, qui n'est pas d'opposition mais de réciprocité : une valeur n'est reconnue comme telle que lorsqu'elle fait l'objet d'une croyance, ou plutôt d'une foi : lorsqu'elle contient la force du sacré. «La valeur ne valorise que dans la mesure où elle sacralise » (p. 179). Il serait donc trompeur de ne voir dans le sacré que l'interdit collectif, le tabou, propre aux sociétés primitives, et dans la valeur l'objet d'une libre adhésion de l'individu des sociétés modernes. Le sacré n'est pas qu'un impératif, il recueille une adhésion collective, il affirme ce qui est bon, juste et saint, il se présente donc comme une valeur, il se manifeste aussi comme désirable (p.190-191). C'est pourquoi Ellul rejette le préjugé selon lequel le choix individuel définirait la situation éthique par excellence, en référence à une morale naturelle. L'homme des sociétés traditionnelles agit tout aussi moralement que l'individu moderne, bien qu'il ne s'autodétermine pas selon des «valeurs" mais se conforme à la volonté collective pour faire ce qui est bien et désirable (p. 190). La "morale de la décision » est une idée romantique, l'expression d'un "devoir être " sécularisé, et qui ne concerne qu'une toute petite partie de la morale vécue : l'essentiel de celle-ci consiste en des actes routiniers et conformistes (p.194). Lorsque l'individu moderne agit selon la coutume morale, il se distingue peu de l'homme traditionnel.

11 Il n'y a donc pas lieu d'établir une discontinuité morale entre les sociétés traditionnelles et les sociétés modernes, mais de considérer le phénomène moral dans ses variétés. Ainsi la vengeance, qui tient une si grande place dans les codes éthiques - et judiciaires traditionnels, est à la fois une exigence du groupe et une obligation individuelle porteuse 
du Bien. L'homme traditionnel qui s'y conforme agit tout aussi moralement qu'un individu moderne qui condamne la vengeance : celle-ci a cessé d'être un devoir moral, son exercice a été retiré aux groupes et réservé à l'État, mais elle est l'une des racines profondes des devoirs moraux qui s'expriment en termes d'honneur, de solidarité ou de fidélité (p. 142-143). Ce point de vue d'Ellul sur les morales du monde l'amène à conclure que celles-ci, qu'elles soient païennes ou chrétiennes - éthiques du clan, de la nation, de la race, de la classe - prescrivent d'agir au nom du même critère moral : bien agir, c'est agir «dans l'intérêt de... ». C'est ainsi que le mensonge, le faux serment, etc. sont des devoirs moraux : il est bien d'agir ainsi dans l'intérêt du frère de clan, de race, de classe, etc. (p. 144).

\section{Le besoin de justification : moralisme et immoralisme}

Ellul interroge alors l'attitude de l'homme face à la morale de la société dans laquelle il vit. Quelle que soit celle-ci, l'homme ne peut jamais être pleinement «moral»: il y a toujours un décalage, voire une opposition, entre la Morale et les Mœurs, entre le devoirêtre et l'être (p. 197). Cet écart tient d'abord à des facteurs sociaux. Leur temporalité est différente : la morale codifie les mœurs d'hier, elle est toujours en retard sur les mœurs d'aujourd'hui, qui influenceront la morale de demain. Leur rationalité est différente : les mœurs sont un phénomène spontané, la morale est toujours plus ou moins construite et organisée. L'écart est dû aussi à des facteurs psychologiques : l'homme tient à justifier ses conduites et il ne peut accepter de n'être jamais à la hauteur des exigences d'une morale qu'il tient pour vraie, pas plus qu'il ne peut accepter son immoralité, parce que cela reviendrait à agir contre ce qu'il reconnaît pour le Bien (p. 207). À l'époque contemporaine, affirme Ellul, cette double impossibilité - être vraiment moral ou s'accepter comme immoral - se manifeste par les attitudes symétriques que sont le moralisme et l'immoralisme. L'un comme l'autre sont des instrumentalisations de la morale à fins de justification, mais aussi de commodité des relations sociales. La justification peut être directe - le moraliste affirme que ce qu'il fait est « bien » parce qu'il le fait - ou indirecte - il se conforme aux commandements moraux pour s'affirmer comme juste face aux injustes. Dans les deux cas, le moraliste cultive sa bonne conscience, il s'évite toute remise en question tout en s'autorisant à mettre autrui en question. Le moraliste se donne aussi un masque social de respectabilité, en s'attendant que les autres fassent de même. Ce conformisme rend prévisibles et calculables les conduites, il permet de juger et classer, c'est un des éléments de la civilité, dont la transgression apparait comme scandale moral (p. 199-200). Ellul range donc dans le moralisme une bonne partie des normes et conventions sociales. Enfin, et particulièrement dans les sociétés individualistes, le moralisme est une affirmation de soi, de l'autodétermination souveraine de l'individu capable de connaître - en lui-même et par sa propre expérience le Bien et le Mal, donc de se faire juge de la Totalité (p. 202). L'effet essentiel du moralisme est alors, en intégrant Dieu dans l'individu autonome - un individu qui se justifie lui-même et devient sa propre valeur suprême - de supprimer le prochain, donc d'abolir toute véritable relation humaine réciproque (p. 203).

L'autojustification de l'immoralisme est jumelle de celle du moralisme dont il n'est même, selon Ellul, qu'un aspect particulier. En récusant la morale qui contrarie sa conduite, l'immoraliste se justifie à ses propres yeux de suivre ses passions auxquelles il ne peut résister. «L'immoraliste libéré de la morale est esclave de ses passions» (p. 204). Et le 
besoin de s'expliquer et de se justifier publiquement - Ellul se réfère évidemment à Gide et à D. H. Lawrence - montre qu'il obéit au même processus intérieur que le moraliste. Une autre forme d'immoralisme, relevant de la commodité sociale, consiste à récuser les préceptes moraux déclinants, vieux jeu, pour suivre et promouvoir les mœurs actuelles de son milieu, le courant sociologique du moment. L'immoralisme d'aujourd'hui prépare ainsi le conformisme de demain, donc un nouveau moralisme (p. 205). Ellul accable les existentialistes de son temps, mais les exemples ne manquent pas depuis lors d'une telle dialectique.

Le besoin de justification est donc un ressort essentiel du phénomène moral : l'homme veut se justifier, avoir raison avec la morale ou contre elle. C'est dans ce cadre qu'Ellul envisage les impasses de l'éthique contemporaine. Les tentatives d'en sortir à partir de la raison conduisent à un amoralisme, qui prétend récuser la morale au nom de vérités supérieures tirées de la Science, avec Marx et Freud notamment. Mais cet apparent dépassement n'est pas celui de la morale, c'est celui d'une morale, la morale bourgeoise du XIX ${ }^{e}$ siècle. De plus, et c'est l'essentiel pour Ellul, il contribue en fait à créer une nouvelle morale (p. 205-206). « Derrière Freud est né un système de morale pragmatique, du comportement et du Normal»(p. 206). Quant au marxisme, il est devenu un moralisme caricatural. La société que les communistes ont voulu créer « est certainement la plus moraliste des sociétés modernes, et elle reprend la bonne vieille morale bourgeoise dans son fond comme dans ses expressions que Karl Marx avait cru liquider » [ ibid.]. En URSS, et plus encore en Chine, les critères moraux applicables à l'« homme socialiste" étaient "des critères de comportement ayant pour but de faciliter une certaine technique politique et sociale»[ibid.]. Ainsi, freudisme et marxisme ont contribué à l'avènement de la "morale technicienne », le système de justification de la « société technicienne » dont Ellul fait la critique dans son œuvre sociologique.

\section{Une morale sans péché : la morale technicienne}

La morale technicienne, en faisant de l'efficacité le motif central du système de valeurs, demande à l'homme d'adhérer moralement à des règles qui ne découlent pas de principes moraux. C'est une morale de comportement dont la problématique est psychologique et sociologique, non «morale» (p. 212). Elle promeut les critères utilitaristes de la société technicienne, et elle répond aux besoins de justification de ses membres en réduisant la tension entre la morale et les mœurs, en formulant «ce qu'il faut faire» dans l'ordre technique. Le Bien technique redéfinit le «Moral» comme "Normal», et conduit à une morale de l'utilité sociale, une «morale sans péché » (p. 198). Dans le contexte des années 1960, Ellul voit cette morale technicienne hégémonique aussi bien à l'Ouest - avec l'application des techniques de "relations humaines » au management des entreprises et à l'optimisation des conduites - qu'à l'Est - l'éthique des pays socialistes devant beaucoup moins aux concepts marxistes qu'aux données techniques - (p. 209). Cette morale est essentiellement comportementale, car le Bien est ce que la technique permet et commande dans le cadre d'une organisation, la technique étant elle-même une façon d'agir . Les techniques psychologiques personnalisent les façons d'agir en raisons d'agir, et permettent d'obtenir bonne volonté et adhésion (p. 213).

Selon Ellul, la morale technicienne découle indirectement de la Réforme. Celle-ci, en désacralisant le monde, a produit un certain indifférentisme spirituel au sociopolitique à l'inverse de l'Église catholique - et conduit la morale chrétienne à promouvoir des 
valeurs techniques de transformation du monde. La morale bourgeoise a été celle de la première phase de la société technicienne, issue de la révolution industrielle. Celle d'aujourd'hui devient purement technicienne, elle tend à éliminer tous les résidus chrétiens de la morale bourgeoise au profit de la technique. Cette dernière devient ellemême une raison d'agir, porteuse d'une promesse de «Mieux » (p. 210-211).

Avec la morale technicienne la norme - le «normal» - rejoint la valeur - le « moral » mais cette morale du normal ne se confond pas tout à fait avec les mœurs, car ses exigences sont définies par des techniciens - statisticiens, cliniciens... - sans qu'aucun principe supérieur ne la transcende : c'est la "moyenne» qui devient souhaitable et désirable, à laquelle l'individu est appelé à s'adapter (p. 214-215). Le Bien et le Mal étant définis de façon expérimentale et pragmatique, ils tendent à se confondre avec la réussite et l'échec, ce qui donne une importance éthique à la performance et au champion : un résultat positif ne se discute pas. La technique est l'instrument de la réussite, et la réussite participe au Bien final (p. 217-218). Plus grand, plus haut, plus fort... Avec sa confiance dans le Futur, la morale technicienne est une morale de l'illimité. «Le Bien apparait alors dans le franchissement de la limite: ce que l'on ne peut pas faire aujourd'hui, on le pourra demain: et cela est bien»(p.221). Ainsi cette morale technicienne, si elle apparait en congruence avec le monde technique qui est le nôtre, tend vers une prévalence de la norme sociale aux dépens des vertus personnelles.

Après avoir présenté l'impossibilité pour l'homme d'échapper à l'impératif moral et l'impossibilité de s'y soumettre vraiment - il ne peut être ni vraiment moral ni vraiment immoral - Ellul s'est donc attaché à montrer qu'il est vain, devant les impasses d'une introuvable morale autonome, naturelle ou universelle, de se fier à la puissance de l'homme lui-même. Le recours à une instance supérieure à la morale, transcendant les représentations humaines du Bien et du Mal, ne peut que faire intervenir une puissance extrinsèque (p. 207). Et c'est à ce recours qu'Ellul consacre la troisième partie de son ouvrage, en affirmant que la tentative de fonder une éthique chrétienne sur la Révélation de la volonté de Dieu est à la fois impossible et nécessaire, comme le montre l'histoire du christianisme.

\section{La morale chrétienne comme produit de l'Histoire}

Le mot « éthique » n'apparait jamais dans le Nouveau Testament, observe-t-il, alors qu'il est courant dans la philosophie hellénistique qui en est contemporaine. De même le mot « devoir » n'y est jamais employé dans un sens moral. Il est question de « marcher selon la volonté de Dieu ", de s'attacher à agir, à vivre, à être selon les commandements, et l'on n'y trouve pas la construction théorique d'une morale (p. 225). Il n'est donc pas possible d'en tirer un «code» formulant une éthique chrétienne sans attenter au jugement de Dieu, alors que le chrétien vit sous le jugement de Dieu: le Bien, dans la Bible, ce n'est jamais un Bien en soi défini comme un idéal, c'est toujours la volonté de Dieu ici et maintenant,sous la forme d'un commandement singulier - «tu», non pas «vous» ou " on" - alors qu'un code éthique formule un Bien qui précède la situation éthique: connaissable par l'homme même s'il lui est inaccessible, analysable et déclinable en types et en normes, et d'une valeur permanente, permettant une cohérence entre hier et aujourd'hui. Or dire que le Bien est la volonté même de Dieu ici et maintenant, c'est reconnaître qu'une éthique construite ne peut exprimer cette volonté en permanence (p. 227-228). Cette illusion n'est au fond guère différente de celle des autres hommes qui 
prétendent, sans Dieu ou contre lui, définir eux-mêmes le Bien et le Mal. Dans le monde judéo-chrétien, elle conduit à ce que la tradition réformée nomme «légalisme», et dont le pharisaïsme est un type caractéristique selon Ellul. Les pharisiens n'étaient pas des "hypocrites» au sens actuel, c'étaient des hommes de bien soucieux de connaître et d'appliquer la Loi, de se «mettre en règle » avec Dieu. Mais là était précisément le mal que leur reprochait Jésus, leur volonté de ne rien demander à Dieu et de ne rien lui devoir (p. 231). Or il ne suffit pas de comprendre la parole de Dieu pour pouvoir l'accomplir. Cette capacité est donnée par le Saint-Esprit, qui souffle où il veut. «Car c'est Dieu qui produit en vous le vouloir et le faire, selon son bon plaisir $»^{1}$, la formule de Paul dont Ellul a tiré le titre de son ouvrage (p. 241). Une vie chrétienne est donc l'œuvre d'une puissance et non l'application d'une norme (p. 247). On ne peut construire une éthique chrétienne sur des modèles, des Saints à imiter, car l'acte ne vaut que par la personne qui l'accomplit, comme un arbre porte ses fruits (p. 244-245). C'est pourquoi l'on peut trouver dans les Évangiles aussi bien « Heureux serez-vous, lorsqu'on vous outragera, qu'on vous persécutera [...] à cause de moi $\aleph^{2}$, lorsque le choix éthique a été fait dans la foi, que "Malheur à vous, scribes et pharisiens hypocrites " $^{3}$ lorsqu'il a été la simple application d'une norme (p. 248-249). Une éthique chrétienne ne peut donc être seulement une morale appliquée, elle est une morale vivante dont le moteur est la foi. Le christianisme est au fond une "Antimorale », car il détruit toutes les morales existantes sans donner naissance à aucune morale qui soit l'expression fidèle de la vérité révélée (p. 251).

Et pourtant le pharisaïsme a été et reste une tentation constante, avec sa recherche d'une sainteté morale, son autoexamen scrupuleux et sa valorisation du mérite, contre la religion du cœur (p. 255). Il y a une séduction de la morale, une préférence pour l'éthique, expression du " phénomène moral » qui se traduit par une pluralité de réponses éthiques, non seulement selon les époques mais au sein même des Églises. On peut s'inspirer d'Augustin, Ambroise, Luther, Calvin, etc., pour la dogmatique, la théologie, mais pas pour leur éthique qui, liée à et exprimant les conditions de leur époque, est par définition dépassée (p. 253-254).

21 Ellul se demande alors comment la question de la conduite a pu venir au premier plan et conduire à la «théologie des œuvres» de l'Église catholique romaine, jusqu'à faire reconnaître par des non-chrétiens la «morale » comme l'essentiel du christianisme (p. 254).

Le cadre moral du christianisme s'est construit « coutumièrement » - au sens de l'Histoire des institutions - au II ${ }^{e}$ siècle, à partir des écrits postpauliniens et des réponses données par les évêques aux questions pratiques posées par les fidèles sur le mariage, l'obéissance à l'empereur, l'usage de l'argent, les spectacles, l'exercice de leur métier, etc. La pénitence, avec son dispositif d'aveu, d'expiation et de réconciliation, est venue répondre à la question posée par l'épître aux Hébreux : celui qui pèche volontairement après avoir reçu le baptême est-il encore chrétien $?^{4}$ Le péché est alors aussi une faute morale, que le sacrifice du Christ n'a pas rachetée par avance (p. 258). C'est au III siècle que la pénitence devient une institution pour des fautes morales comme l'adultère et la fornication, et que les théologiens commencent à importer dans le christianisme les questions traitées par les philosophes grecs, constituant ainsi le cadre d'une morale chrétienne. Les tensions hérétiques de l'époque apparaissent souvent à partir des questions de morale. Théologiens et apologètes introduisent donc dans le christianisme les principes moraux du stoïcisme qui vont bientôt en constituer l'essentiel, alors que les anachorètes et les ermites tenteront de maintenir une tradition éthique purement biblique (p. 259). Ce 
processus s'institutionnalise au siècle suivant sous Constantin: c'est aux autorités ecclésiastiques qu'il revient de définir le bien agir, objectivant ainsi une éthique dans laquelle le lien entre la foi et l'œuvre est rompu. Dès lors, la part de l'éthique ne cesse de croître dans les siècles qui suivent, les problèmes étant systématisés sous l'angle moral en casuistique et en philosophie. Trois raisons à cela selon Ellul. En premier lieu, dans une Église établie, dont la majorité des membres sont des païens convertis administrativement et politiquement sans avoir vécu une expérience spirituelle de la grâce, chacun veut connaître ses devoirs et obligations : la cure d'âme devient un contrôle des conduites, faute d'agir sur les consciences (p.261). Puis, la société est devenue chrétienne, la "Chrétienté », dans laquelle le christianisme est une affaire collective : les institutions doivent être chrétiennes, ce qui se réalise par une christianisation de l'existant. Une morale provenant de sources diverses - non plus seulement grécoromaines mais barbares - est déclarée chrétienne, la Scolastique tentant une synthèse entre la morale de la société («morale sociologique») et la doctrine du Saint-Esprit (p. 262-263). Enfin, l'Église s'est superposée à la société et prétend la diriger : la « morale chrétienne » doit s'appliquer à tous. Mais cette morale qui s'est constituée entre le IV et le XIII ${ }^{e}$ siècle est une morale sociologique parmi d'autres morales humaines, qui ne peut se prétendre inspirée par le Saint-Esprit (p. 264-265). Il était légitime pour les chrétiens devouloir transcrire l'exigence de la foi en termes éthiques, et il était inévitable qu'ils n'y parviennent pas, suivant en fait la morale de leur monde. « Mais qu'ils élaborent pour des raisons du monde, dans les conditions du monde, et sous l'impulsion du monde une morale qu'ils baptisent chrétienne, cela est une imposture » (p. 265).

Un seuil décisif est franchi avec la Réforme, bien que les premiers réformateurs n'aient pas, malgré les apparences, élaboré une éthique à partir de leur théologie. Ellul souligne la singularité de la réforme luthérienne. Il y avait déjà eu plusieurs réformations majeures, dont la réforme grégorienne, «mais la voie choisie était toujours morale et institutionnelle, jamais théologique et spirituelle. L'importance prise par la morale dans l'Église est en effet bien mise en lumière par le type même des réformes entreprises dans la chrétienté depuis le $\mathrm{VI}^{\mathrm{e}}$ siècle jusqu'au XVI ${ }^{\mathrm{e}}$ siècle » (p. 270). Elles visaient l'amélioration morale de la vie des chrétiens - surtout du clergé - et le perfectionnement des institutions de l'Église. C'est par la morale sociale que l'on voulait atteindre le cœur des chrétiens. Mais cela supposait un appui sur une autorité extérieure, institutionnelle, juridique, et renforçait son pouvoir. Ce qui est nouveau avec Luther, c'est la volonté de réformer l'Église en partant du cœur des croyants, par la théologie et la vie spirituelle, la morale devant suivre (p. 270-271).

Cependant, cette réaffirmation du salut par la grâce et par le moyen de la foi a mis en question tout le système moral de l'Église (p. 270), et les pasteurs réformés ont dû à leur tour répondre à la nécessité de formuler une éthique. Les théologiens réformateurs n'ayant pas eux-mêmes tiré les conséquences éthiques de leur dogmatique, les pasteurs vont se référer directement à la Bible, tant aux textes moraux de l'Ancien Testament qu'aux exhortations des Épitres. Cela a produit dans la plupart des cas une éthique figée, alors que l'inscription directe des commandements divins dans la vie concrète est impossible du fait de l'évolution constante que connaît la société humaine (p. 274). Ellul mentionne en particulier «la tentative surhumaine et impossible des Puritains [qui] fut d'inscrire une conduite monastique dans le monde, mais cela supposait alors que le monde n'évolue pas et que la situation fixée une fois reste identique à elle-même » (ibid.). Il y avait là selon lui une confusion entre la vérité théologique de la Révélation, qui est 
permanente et intangible, et le commandement moral qui ne peut avoir le même caractère. Le résultat en fut une morale "inconsciente ", bâtie sur l'illusion d'une inscription du commandement de Dieu dans la vie comme une loi, « ce qui est le comble comme conséquence de la théologie de la grâce ! » (ibid.).

Melanchton a, parmi les premiers, contribué à séparer la dogmatique de la morale, mais c'est plus tard, au $\mathrm{xVIII}^{\mathrm{e}}$ siècle, que l'éthique s'est constituée comme domaine complètement distinct, avec les Lumières et le piétisme. Les unes ont pris pour objet la conscience morale de l'homme naturel, l'autre la psychologie de l'homme régénéré par la grâce. Kant n'a fait que pousser à l'extrême cette autonomie de la morale. Le résultat de cette évolution a été dans la société l'identification du christianisme à une morale, celle de la classe montante puis dominante - la "morale chrétienne " a été assimilée à la «morale bourgeoise » - cette dernière étant ensuite contestée par une « éthique sociale chrétienne » puisant à de nouvelles sources. Il n'a jamais été possible en effet, remarque Ellul, de construire une éthique chrétienne sans lui incorporer des apports étrangers à la Révélation : stoïcisme, aristotélisme, rationalisme, marxisme... (p. 267).

\section{Impossibilité et nécessité d'une éthique chrétienne} a été perdue, car ces tentatives de définir des comportements «objectivement bons », en conjuguant la Révélation avec d'autres sources, transforment le Personnel en Impersonnel: l'aléa de la relation personnelle avec Dieu, ce qu'elle peut avoir de redoutable pour l'individu et de dangereux pour l'ordre de l'Église, fait place à un système de normes préétablies donnant la signification de la relation de l'homme en soi avec Dieu (p. 268). Dès lors que la volonté de Dieu est codifiée en ordre moral, il suffit de s'y conformer pour accomplir cette volonté : ni l'expression ni la réception personnelle de la Parole divine ne sont nécessaires (ibid.). Dans le cadre de l'Église romaine, la conséquence théologique de la morale ecclésiastique ne pouvait être que le salut par les œuvres: il s'agit d'agir selon la règle, avec l'« aide » de l'Église. La Réforme a bien réaffirmé que le salut n'est accordé que par la grâce, sans que les œuvres y contribuent, mais l'accomplissement des œuvres reste indispensable, tant pour la vie de la Chrétienté que pour la conduite individuelle. Même si les Réformateurs ont échoué dans l'entreprise de la formulation d'une éthique, celle-ci n'en est pas moins nécessaire, et c'est sur cette affirmation que Jacques Ellul veut conclure.

Une bonne théologie à elle seule ne peut guider l'engagement dans l'action, car celle-ci ne pourra que suivre les courants sociologiques du moment. La seule référence à une théologie transcendante peut laisser l'homme désemparé dans le monde, avec trois justifications qui brisent le lien entre la rénovation christique et l'action: l'illusion du "piétisme spirituel» par lequel l'homme peut se croire sous la conduite directe et permanente du Saint-Esprit ; l'indifférentisme moral aux problèmes du monde considérés comme sans importance ; l'engagement dans les problèmes du monde selon les options morales et politiques des autres hommes, sans référence au christianisme qui n'aurait rien à dire sur eux (p. 274). La formulation d'une éthique dans l'Église est donc indispensable, puisque le commandement de Dieu ne peut s'inscrire directement dans la vie. L'échec des théologiens réformateurs provient de ce qu'ils ont laissé les pasteurs et les fidèles puiser directement dans la Bible un guide de conduite prenant le texte à la lettre (ibid.). Si l'éthique est nécessaire, ce n'est pas pour offrir des solutions à chaque 
problème, mais pour rappeler au chrétien que sa conduite spécifique est la conséquence indispensable de sa foi. Elle est là pour circonscrire les limites du commandement de Dieu et de l'action correspondante de l'homme, selon une formule reprise de Karl Barth (p. 276). Une éthique formulée par l'Église exige un renouvellement constant puisqu'elle n'est pas l'application immédiate et immuable du commandement. Une éthique suit les circonstances concrètes et changeantes de la vie, ou bien elle n'est pas une éthique (p. 275). De plus, l'exigence qu'elle proclame est exhortative et non dogmatique, elle est un appel, qui est entendu par l'individu qui le prend au sérieux à cause de sa foi, et parce que le Saint-Esprit, à cause de sa foi, lui a accordé une force suffisante pour vivre cette exigence dans son comportement face aux circonstances de sa vie (p. 280). Elle a aussi une fonction critique : c'est la confrontation à l'exigence éthique qui amène le chrétien à se demander constamment s'il est vraiment « dans la foi » et non plus « sous la loi » (p. 281). La foi ne nous libère pas de la loi pour nous permettre de ne pas l'accomplir, mais nous conduit au contraire à "prendre au sérieux le relatif du monde » en continuité avec le dessein divin. Ellul illustre cette position classique face à l'« antinomisme $»^{5}$ par l'exemple évangélique de la dîme : le paiement de la dîme était une exigence de la loi ; la vie dans la foi libère le chrétien de cette exigence, non pas qu'elle lui évite d'avoir à payer la dîme, mais elle le place devant une exigence plus haute, celle de consacrer tous ses biens à Dieu, dont la dîme n'est qu'une infime partie (p. 282). La conclusion d'Ellul reprend la formule de Reinhold Niebuhr : l'éthique reste pour le chrétien une « impossible possibilité » (p. 294). Ce qui rend possible et légitime le discours éthique, comme le propose Bonhoeffer, c'est que Dieu lui-même a exprimé sa volonté sous la forme d'un commandement. Il faut entendre la révélation passée, celle de l'Écriture, pour en tirer un enseignement au présent (p. 290). Cette parole, que le Saint-Esprit rend vivante, présente et actuelle, n'a pas en elle-même une signification éthique, mais fait naître en nous une exigence éthique (p. 291). Celle-ci rencontre alors trois limites.

La première est qu'une éthique chrétienne ne peut prétendre définir objectivement le Bien ni exprimer la volonté même de Dieu: elle se reconnaît relative, elle admet que «toute recherche autonome de la connaissance du Bien et du Mal est exclue » selon les mots de Karl Barth (p. 292).

Par suite, elle ne peut pas plus "posséder » le Bien que le connaître. Le chrétien n'est jamais « en règle » avec Dieu à la manière des pharisiens, il doit renoncer à l'autonomie (p. 292-293).

31 Enfin elle ne peut être impérative puisque, bien que légitime et nécessaire, elle reste impossible. Ellul en avait fait son point de départ: la morale n'est qu'humaine, elle appartient au monde, c'est-à-dire qu'elle se situe entre la chute de l'homme et le retour du Christ. Elle ne peut prétendre ni à la connaissance de la volonté de Dieu - comme si la chute n'avait pas eu lieu - ni à la perfection- comme si le retour du Christ avait déjà eu lieu (p. 293).

32 À strictement parler, il ne peut donc s'agir de promouvoir une éthique chrétienne mais une « éthique pour les chrétiens » (ibid.), ceux-ci devant résister à la tentation de vouloir transformer l'extraordinaire en ordinaire, réduire l'exigence divine à hauteur d'homme. 


\section{La portée anthropologique de la critique théologique d'Ellul} polémique a évidemment perdu de son actualité, car il suffit de rappeler le contexte de l'époque - confrontation idéologique entre les deux blocs, vitalité du christianisme social, influence des intellectuels de gauche sur l'esprit du temps - pour faire ressortir la distance historique qui nous en sépare. Il reste que ses propositions concernant le phénomène moral gardent toute leur force- d'une part, l'homme ne peut trouver en luimême un fondement universel à sa morale sinon un besoin de justification auquel tous les groupes humains répondent à leur façon; d'autre part, l'efficacité technique ne peut constituer le «motif principal» d'un système de valeurs - et que leur portée dépasse largement le champ théologique.

première proposition peut être mise en regard de celle que faisait Marcel Gauchet à la fin des années 1990 : la sortie de la religion n'aboutit pas à la disparition du religieux mais à la persistance d'un "religieux sans la religion », car « l'expérience humaine est depuis toujours une expérience de l'altérité de soi » qu'il faut bien interpréter d'une façon ou d'une autre (Gauchet, p. 200). Si Gauchet faisait l'hypothèse d'un « noyau anthropologique du religieux" (ibid., p. 205), Ellul faisait en quelque sorte celle d'un noyau anthropologique de la morale, consistant en un besoin de justification indissociable de la condition humaine. Dans sa vision théologique, il s'agissait de la condition de l'homme déchu, " autonome ", ayant à définir lui-même le Bien et le Mal et devant donc se justifier à ses propres yeux. Mais les anthropologues, sociologues et psychologues pourraient reprendre cette proposition sans adhérer au postulat chrétien de la Chute : ce qu'exprime le phénomène moral, c'est que l'expérience de soi n'est pas seulement cognitive et affective, elle est aussi nécessairement une expérience éthique. Le « sentiment de soi» n'inclut pas seulement la perception d'un écart entre ce que l'on pense et ce que l'on ressent (Damasio, 1999), mais entre ce que l'on doit faire et ce que l'on fait.

(ailleurs, la critique d'Ellul est en résonance avec les thèses que des auteurs américains comme Philip Rieff et Christopher Lasch ont développées dans les années qui ont suivi, et qui en apparaissent aujourd'hui complémentaires (Rieff, 1966, Lasch, 1979). Le premier affirmait l'impossibilité pour les sociétés occidentales contemporaines de se passer de toute transcendance pour fonder les conduites humaines, le second critiquait l'influence du protestantisme libéral dans l'appauvrissement des bases du sentiment de soi. Tous deux dénonçaient, comme Ellul, la morale de l'illimitation et l'éthique "thérapeutique " qui résultait selon eux d'une psychologisation de la société et de la monopolisation du Bien par les experts. Cette éthique thérapeutique peut apparaitre comme une des modalités de la morale technicienne que dénonçait Ellul, une morale sans péché qui transforme les dilemmes moraux en troubles psychiques ou en dysfonctionnements à traiter, et qui valorise la performance.

Quant à la dimension psychologique du phénomène moral, on peut observer aussi que, dès les années 1970, les cognitivistes ont tenté d'en rendre compte, à la suite de Robert Solomon, en faisant de l'expérience affective-émotionnelle de l'individu le fondement de ses jugements, y compris moraux. Solomon répondait à la position de Camus sur l'absurdité de la vie, en affirmant que nos émotions sont des « jugements normatifs » : la vie n'est pas absurde si nous acceptons de lui trouver un sens dans l'expérience de nos

Archives de sciences sociales des religions, 168 | 2014 
passions - émotions, humeurs, désirs (Solomon, IX, XV, 126) ${ }^{6}$. Alors qu'Ellul affirmait la nécessité de dépasser le leurre du rationalisme par l'acceptation d'une volonté transcendante, l'écoute d'une voix intérieure qui nous dit: «tu», Solomon proposait de chercher cette puissance symbolique dans une expérience intérieure sécularisée. Il contredisait ainsi le dualisme rationaliste qui faisait de la dimension affectiveémotionnelle une perturbation du jugement. Il contestait aussi le freudisme - du moins son courant dominant, l'Ego Psychology - qui, pour lui comme pour Ellul, déresponsabilisait l'individu de ses passions en situant celles-ci dans un "ça » extérieur au «moi». Solomon affirmait au contraire que nous sommes responsables de nos passions, elles-mêmes sources de nos propres jugements de réalité et de valeur, «le tribunal suprême de la conscience » (ibid., XVII) ${ }^{7}$.

Si la critique de la morale par Ellul dans Le Vouloir et le Faire, qui se voulait avant tout théologique, peut intéresser aussi aujourd'hui le public des scienceshumaines et sociales, cela tient à la position singulière de cet auteur dans le champ intellectuel de son temps, qui été rappelée plus haut: ses positions théologiques ne prennent tout leur sens que dans leur rapport avec le volet sociologique de son œuvre. On peut donc avoir à l'esprit sa proposition anthropologique concernant l'universalité du besoin de justification lorsqu'on aborde les travaux les plus contemporains qui cherchent à rendre compte du sentiment de soi, et envisager avec lui que la dimension éthique puisse en être constitutive.

\section{BIBLIOGRAPHIE}

DAMASIO Antonio, 1999, The Feeling of what Happens : Body and Emotion in the Making of Consciousness, New York, Harcourt, Éd. fr.

-, 1999, Le sentiment même de soi. Corps, émotions, conscience, Paris, Odile Jacob.

Ellul Jacques, 2013 (1964), Le Vouloir et le Faire. Une critique théologique de la morale, Genève, Labor et Fides.

GAUCHET Marcel, 1998, « Essai de psychologie contemporaine. II. L'inconscient en redéfinition », Le Débat, $\mathrm{n}^{\circ} 100$, p. 189-206.

LASCH Christopher, 1979, The Culture of Narcissism : American Life in an Age of Diminishing Expectations, New York, Norton. Éd. fr.

-, 2006 (2000), La Culture du narcissisme : la vie américaine à un âge de déclin des espérances, Paris, Flammarion.

RIEFF Philip, 2006 (1966), The Triumph of The Therapeutic. Uses of Faith after Freud, Wilmington (DE), ISI books. 


\section{NOTES}

1. Philippiens $2: 13$.

2. Matthieu $5: 11$.

3. Matthieu $23: 15$; Luc $11: 14$.

4. Hébreux $10: 26$.

5. L'« antinomisme " désignait pour Luther une interprétation trop extrême de la justification par la foi seule selon laquelle, avec le Christ, l'obéissance à la Loi n'est plus une condition de salut. Si c'était le cas, en effet, une telle conception « légale » ou contractuelle du salut ne serait qu'un retour au salut par les œuvres. L'antinomisme n'implique pas nécessairement un laxisme moral, il peut consister en une attention exclusive apportée à "l'infusion de la grâce ", l'action intime du Saint-Esprit comme seul guide de conduite, l'indifférence à la recherche d'une conduite de vie vertueuse, une tendance au " quiétisme ».

6. . "Thus I suggest that the passions are the meaning of life. It is because we are moved, because we feel, that life has a meaning " (IX). «Our passions - our emotions, moods and desires - define us, our selves, and the world we live in " (XV). "An emotion is an evaluative (or a "normative") judgment, a judgment about my situation and about myself and/or about all other people » (p. 126).

7. . " the high court of consciousness ».

\section{AUTEURS}

\section{PIERRE PRADES}

Docteur en sociologie, chercheur rattaché au Sophiapol, Université Paris Ouest-Nanterre-La Défense, pprades@club-internet.fr 